\title{
EIGENVALUE PROBLEMS FOR SINGULAR ODES
}

\author{
DONAL O'REGAN \\ Department of Mathematics, National University of Ireland, Galway, Ireland \\ and ALEKSANDRA ORPEL \\ Faculty of Mathematics and Computer Science, University of Lodz, Poland \\ e-mail: orpela@math.uni.lodz.pl
}

(Received 23 December 2009; accepted 28 June 2010; first published online 9 December 2010)

\begin{abstract}
We investigate eigenvalue intervals for the Dirichlet problem when the nonlinearity may be singular at $t=0$ or $t=1$. Our approach is based on variational methods and cover both sublinear and superlinear cases. We also study the continuous dependence of solutions on functional parameters.
\end{abstract}

2010 Mathematics Subject Classification.

1. Introduction. We investigate the Dirichlet problem

$$
\begin{aligned}
& -u^{\prime \prime}=f(t, u)+\lambda g(t, u) \text { a.e. in }(0,1) \\
& u(0)=u(1)=0
\end{aligned}
$$

under the following assumptions:

(H1) there exists $d>0$ such that $f, g:(0,1) \times(-\varepsilon, d+\varepsilon) \rightarrow R$ are Caratheodory functions, $f+\lambda g$ is nondecreasing with respect to the second variable in $(-\varepsilon, d+\varepsilon), \varepsilon$ is given positive number.

(H2) $\max _{u \in[0, d]}|f(t, u)+\lambda g(t, u)|$ belongs to $L^{2}(0,1) ; f(t, 0)+\lambda g(t, 0) \neq 0$.

In the literature there are many papers devoted to singular second-order differential equations (see $[\mathbf{1}, \mathbf{2}, \mathbf{5}, \mathbf{8}, \mathbf{9}]$ ). Most papers discuss the case when the nonlinearity is positive in a certain neighbourhood of zero and it is sublinear with respect to the second variable at infinity. In this paper, we consider (1) where the right-hand side may be singular at $t=0$ or $t=1$. Moreover, our results will cover both sublinear and superlinear cases at zero and/or at infinity. Our approach is based on assumptions on the nonlinearity in the interval $(-\varepsilon, d+\varepsilon)$ only (see Section 2). Assumptions (H1) and (H2) allow us to obtain an existence result for our problem in each nonempty subset $X_{\lambda}$ of $H_{0}^{1}(0,1) \cap H^{2}(0,1)$ such that:

(i) $X_{\lambda}$ has property (D), namely for each $u \in X_{\lambda}$ there exists $\bar{u} \in X_{\lambda}$ such that

$$
\begin{aligned}
& -\bar{u}^{\prime \prime}=f(t, u)+\lambda g(t, u) \text { a.e. in }(0,1) \\
& \bar{u}(0)=\bar{u}(1)=0
\end{aligned}
$$

(ii) for each $u \in X_{\lambda}, u(t) \in[0, d]$ for all $t \in[0,1]$.

In the next section we shall give two examples of $X_{\lambda}$ satisfying (i) and (ii). 
We shall apply variational methods (as in $[4,6,7]$ ), thus equation (1) will be treated as the Euler-Lagrange equation of the integral functional $J_{\lambda}: X_{\lambda} \rightarrow R$ defined as follows:

$$
J_{\lambda}(u):=\int_{0}^{1} \frac{1}{2}\left|u^{\prime}(t)\right|^{2}-H(t, u(t), \lambda) d t,
$$

where $H(t, u, \lambda):=\int_{0}^{u}(f(t, r)+\lambda g(t, r)) d r$ for each $(t, u) \in(0,1) \times[0, d]$ and $\lambda \in R$. Our first task is to describe the numerical characterization of solutions by a minimizing sequence of $J_{\lambda}$ (Theorem 5). Next we apply this result to prove the existence of solution for (1) in the set $X_{\lambda}$. To give the explicit definition of $X_{\lambda}$ we use additional assumptions e.g. $(\mathrm{H} 3)$ or $(\mathrm{H} 4)$, which describe the eigenvalue intervals for our problem. In the last section we discuss the continuous dependence of solutions of (1) on functional parameters. Precisely we show that the sequence of solutions $\left(u_{m}\right)_{m=1}^{\infty}$ (corresponding to parameters $\left(v_{m}\right)_{m=1}^{\infty}$ ) tends uniformly (up to a subsequence) to a certain solution $u_{0}$ of the limit problem (corresponding to parameter $v_{0}$ ) provided that $\left(v_{m}\right)_{m=1}^{\infty}$ tends to $v_{0}$ a.e. in $(0,1)$. We also investigate the asymptotic behaviour of the solution of the limit problem.

\section{Existence of $X_{\lambda}$.}

EXAMPLE 1. (with positive right-hand side of the equation) Assume (H1), (H2) and

(H3) $f+\lambda g \geq 0$ in $(0,1) \times[0, d]$ and the following estimations hold

$$
0<\int_{0}^{1}(f(t, d)+\lambda g(t, d)) d t \leq 4 d
$$

or

$$
0<\int_{0}^{1} t(1-t)(f(t, d)+\lambda g(t, d)) d t \leq d .
$$

Then we define $X_{\lambda}$ as follows:

$$
X_{\lambda}:=\left\{u \in H_{0}^{1}(0,1) \cap H^{2}(0,1), 0 \leq u(t) \leq d \text { in }[0,1]\right\} .
$$

To prove that $X_{\lambda}$ satisfies (i) and (ii) we fix $u \in X_{\lambda}$ and consider

$$
\bar{u}(t)=\int_{0}^{1} G(t, s)(f(s, u(s))+\lambda g(s, u(s))) d s \text { for all } t \in[0,1],
$$

where $G:[0,1] \times[0,1] \rightarrow[0,+\infty)$ is Green's function

$$
G(s, t):= \begin{cases}s(1-t) & \text { for } 0 \leq s \leq t \\ t(1-s) & \text { for } t \leq s \leq 1 .\end{cases}
$$

It is clear that

$$
\begin{aligned}
& -\bar{u}^{\prime \prime}=f(t, u)+\lambda g(t, u) \text { a.e. in }(0,1) \\
& \bar{u}(0)=\bar{u}(1)=0
\end{aligned}
$$


Moreover, in the case when (2) holds we get

$$
\begin{aligned}
0 & \leq \bar{u}(t) \leq t(1-t) \int_{0}^{1}(f(s, u(s))+\lambda g(s, u(s))) d s \\
& \leq \frac{1}{4} \int_{0}^{1}(f(s, u(s))+\lambda g(s, u(s))) d s \leq d .
\end{aligned}
$$

In the case when (3) holds we obtain

$$
0 \leq \bar{u}(t) \leq \int_{0}^{1} s(1-s)(f(s, u(s))+\lambda g(s, u(s))) d s \leq d .
$$

In both cases $0 \leq \bar{u}(t) \leq d$ in $[0,1]$.

REMARK 2. If $g>0$ and $f(\cdot, d), g(\cdot, d)$ belong to $L^{1}(0,1)$ then conditions (H3) can be rewritten as follows:

$$
\frac{-\int_{0}^{1} f(t, d) d t}{\int_{0}^{1} g(t, d) d t}<\lambda \leq \frac{4 d-\int_{0}^{1} f(t, d) d t}{\int_{0}^{1} g(t, d) d t}
$$

or

$$
\frac{-\int_{0}^{1} t(1-t) f(t, d) d t}{\int_{0}^{1} t(1-t) g(t, d) d t}<\lambda \leq \frac{d-\int_{0}^{1} t(1-t) f(t, d) d t}{\int_{0}^{1} t(1-t) g(t, d) d t}
$$

EXAMPLE 3. Suppose that (H1) and (H2) hold and assume

(H4)

$$
\int_{0}^{1} \max _{u \in[0, d]}|f(t, u)+\lambda g(t, u)| d t \leq 4 d
$$

or

$$
\int_{0}^{1} t(1-t) \max _{u \in[0, d]}|f(t, u)+\lambda g(t, u)| d t \leq d .
$$

In the case when the right-hand side of (1) can change sign we can show that the set

$$
X_{\lambda}:=\left\{u \in H_{0}^{1}(0,1) \cap H^{2}(0,1),|u(t)| \leq d \text { in }[0,1]\right\}
$$

has property (D) and show the existence of solution of (1) in $X_{\lambda}$. Of course then the definition of $X_{\lambda}$ gives us no information about sign of solution. I keep working on this case. 
3. Variational principle for a minimizing sequence. We start with the auxiliary property of the domain $X_{\lambda}$ of the functional $J_{\lambda}$.

REMARK 4. Since for each $u \in X_{\lambda}$ there exists $\bar{u} \in X_{\lambda}$ such that (D) holds, we can state that

$$
v(t):=\bar{u}^{\prime}(t) \text { a.e. in }(0,1)
$$

belongs to $H^{1}(0,1)$ and

$$
-v^{\prime}(t) \in \partial_{u} H(t, \bar{u}(t), \lambda) \text { a.e. in }(0,1)
$$

which follows from property (D) and the convexity of $H$ with respect to the second variable. $\left(\partial_{u} H\right.$ denotes the subdifferential of $H(t, \cdot, \lambda)$.)

From Remark 4 and the properties of the Fenchel transform we prove the approximation of a solution by a minimizing sequence of the action functional $J_{\lambda}$.

THEOREM 5. Assume that (H1) and (H2) hold. Let $X_{\lambda}$ be an nonempty set satisfying (i) and (ii). For each sequence $\left(u_{m}\right)_{m \in N} \subset X_{\lambda}$ minimizing $J_{\lambda}: X_{\lambda} \rightarrow R$ there exists sequence $\left(v_{m}\right)_{m \in N} \subset H^{1}(0,1)$ such that

$$
-v_{m}^{\prime}(t) \in \partial_{u} H\left(t, u_{m}(t), \lambda\right)=\left\{f\left(t, u_{m}(t)\right)+\lambda g\left(t, u_{m}(t)\right)\right\} \text { a.e. in }(0,1)
$$

and

$$
\left.\lim _{m \rightarrow \infty} \int_{0}^{1} \frac{1}{2}\left|v_{m}(t)\right|^{2}+\frac{1}{2}\left|u_{m}^{\prime}(t)\right|^{2}-u_{m}^{\prime}(t) v_{m}(t)\right) d t=0 .
$$

Proof. Let us note that $J_{\lambda}$ is bounded from below in $X_{\lambda}$. Indeed, for each $u \in X_{\lambda}$

$$
\begin{aligned}
J_{\lambda}(u) & =\int_{0}^{1} \frac{1}{2}\left|u^{\prime}(t)\right|^{2}-H(t, u(t), \lambda) d t \geq-\int_{0}^{1} \int_{0}^{u(t)}(f(t, r)+\lambda g(t, r)) d r d t \\
& \geq-\int_{0}^{1} u(t) \max _{u \in[0, d]}|f(t, u)+\lambda g(t, u)| d t \geq-d \int_{0}^{1} \max _{u \in[0, d]}|f(t, u)+\lambda g(t, u)| d t,
\end{aligned}
$$

so that $-\infty<$ Min $:=\inf _{u \in X_{\lambda}} J_{\lambda}(u)<+\infty$. This assertion means that for a given $\varepsilon>0$ there exists $m_{0} \in N$ such that for all $m \geq m_{0}, J_{\lambda}\left(u_{m}\right)<\varepsilon+\operatorname{Min}$. Since $\left(u_{m}\right)_{m \in N} \subset X_{\lambda}$, condition (i) guarantees the existence of $\left(\bar{u}_{m}\right)_{m \in N} \subset X_{\lambda}$ such that

$$
\begin{aligned}
-\bar{u}_{m}^{\prime \prime}(t) & =f\left(t, u_{m}(t)\right)+\lambda g\left(t, u_{m}(t)\right) \text { a.e. in }(0,1) \\
\bar{u}_{m}(0) & =\bar{u}_{m}(1)=0 .
\end{aligned}
$$

Let us define $\left(v_{m}\right)_{m \in N} \subset H^{1}(0,1)$ as follows:

$$
v_{m}(t):=\bar{u}_{m}^{\prime}(t) \text { a.e. in }(0,1) .
$$

Then we get

$$
\begin{aligned}
-v_{m}^{\prime}(t) & \in \partial_{u} H\left(t, u_{m}(t), \lambda\right) \\
& =\left\{f\left(t, u_{m}(t)\right)+\lambda g\left(t, u_{m}(t)\right)\right\} \text { a.e. in }(0,1) .
\end{aligned}
$$

Thus (8) follows. 
Coming to the second part of the proof we infer, by the Fenchel equality for $L^{2}(0,1) \ni u \mapsto \int_{0}^{1} H(t, u(t), \lambda) d t$, that for each $m \geq m_{0}$

$$
\operatorname{Min}+\varepsilon>J_{\lambda}\left(u_{m}\right)=\int_{0}^{1} H^{*}\left(t,-v_{m}^{\prime}(t), \lambda\right) d t+\int_{0}^{1} u_{m}(t) v_{m}^{\prime}(t) d t+\int_{0}^{1} \frac{1}{2}\left|u_{m}^{\prime}(t)\right|^{2} d t
$$

where $H^{*}(t, v, \lambda):=\sup _{u \in R}(u v-H(t, u, \lambda))$ for all $(t, v, \lambda) \in(0,1) \times R \times R$.

Moreover, the definition of the Fenchel conjugate gives

$$
\begin{aligned}
\text { Min } & =\inf _{u \in X_{\lambda}} J_{\lambda}(u) \leq \int_{0}^{1} \frac{1}{2}\left|u^{\prime}(t)\right|^{2} d t-\int_{0}^{1} H(t, u(t), \lambda) d t \\
& \leq \int_{0}^{1} \frac{1}{2}\left|u^{\prime}(t)\right|^{2} d t+\int_{0}^{1} H^{*}\left(t,-v_{m}^{\prime}(t), \lambda\right) d t-\int_{0}^{1} u^{\prime}(t) v_{m}(t) d t
\end{aligned}
$$

for all $u \in X_{\lambda}$ and further, we get for all $m \in N$

$$
\begin{aligned}
\text { Min } \leq & \inf _{u \in X_{\lambda}}\left[\int_{0}^{1} \frac{1}{2}\left|u^{\prime}(t)\right|^{2} d t+\int_{0}^{1} H^{*}\left(t,-v_{m}^{\prime}(t), \lambda\right) d t\right. \\
& \left.-\int_{0}^{1} u^{\prime}(t) v_{m}(t) d t\right] \\
= & \int_{0}^{1} H^{*}\left(t,-v_{m}^{\prime}(t), \lambda\right) d t-\sup _{u \in X_{\lambda}}\left[\int_{0}^{1} u^{\prime}(t) v_{m}(t) d t-\int_{0}^{1} \frac{1}{2}\left|u^{\prime}(t)\right|^{2} d t\right] .
\end{aligned}
$$

Now we show that

$$
\sup _{u \in X_{\lambda}}\left[\int_{0}^{1} u_{m}^{\prime}(t) v_{m}(t) d t-\int_{0}^{1} \frac{1}{2}\left|u_{m}^{\prime}(t)\right| d t\right]=\int_{0}^{1} \frac{1}{2}\left|v_{m}(t)\right| d t
$$

Indeed, since $v_{m}(t):=\bar{u}_{m}^{\prime}(t)$ a.e. in $(0,1)$ for certain $\bar{u}_{m} \in X_{\lambda}$, we can see that

$$
\begin{aligned}
\int_{0}^{1} \frac{1}{2}\left|v_{m}(t)\right| d t & =\int_{0}^{1} \bar{u}_{m}^{\prime}(t) v_{m}(t) d t-\int_{0}^{1} \frac{1}{2}\left|\bar{u}_{m}^{\prime}(t)\right| d t \\
& \leq \sup _{u \in X_{\lambda}}\left[\int_{0}^{1} u^{\prime}(t) v_{m}(t) d t-\int_{0}^{1} \frac{1}{2}\left|u^{\prime}(t)\right| d t\right] \\
& \leq \sup _{z \in L^{2}(0,1)}\left[\int_{0}^{1} z(t) v_{m}(t) d t-\int_{0}^{1} \frac{1}{2}|z(t)| d t\right] \\
& =\int_{0}^{1} \frac{1}{2}\left|v_{m}(t)\right| d t
\end{aligned}
$$

Therefore (13) leads to the conclusion that for all $m \in N$

$$
\operatorname{Min} \leq \int_{0}^{1} H^{*}\left(t,-v_{m}^{\prime}(t), \lambda\right) d t-\int_{0}^{1} \frac{1}{2}\left|v_{m}(t)\right|^{2} d t
$$


Finally (12) and (15) imply

$$
\begin{aligned}
0 \leq & \left(\int_{0}^{1} \frac{1}{2}\left|u_{m}^{\prime}(t)\right|^{2} d t+\int_{0}^{1} \frac{1}{2}\left|v_{m}(t)\right|^{2} d t-\int_{0}^{1} u_{m}^{\prime}(t) v_{m}(t) d t\right) \\
= & \left\{\int_{0}^{1} \frac{1}{2}\left|v_{m}(t)\right|^{2} d t-\int_{0}^{1} H^{*}\left(t,-v_{m}^{\prime}(t), \lambda\right) d t\right\} \\
& +\int_{0}^{1} \frac{1}{2}\left|u_{m}^{\prime}(t)\right|^{2} d t+\int_{0}^{1} u_{m}(t) v_{m}^{\prime}(t) d t+\int_{0}^{1} H^{*}\left(t,-v_{m}^{\prime}(t), \lambda\right) d t \\
\leq & - \text { Min }+ \text { Min }+\varepsilon=\varepsilon
\end{aligned}
$$

for all $m \geq m_{0}$. Since $\varepsilon>0$ was arbitrary, we get

$$
\lim _{m \rightarrow \infty}\left(\int_{0}^{1} \frac{1}{2}\left|u_{m}^{\prime}(t)\right|^{2} d t+\int_{0}^{1} \frac{1}{2}\left|v_{m}(t)\right|^{2} d t-\int_{0}^{1} u_{m}^{\prime}(t) v_{m}(t) d t\right)=0 .
$$

\section{Existence result.}

THEOREM 6. Assume hypotheses (H1)-(H2) and suppose that $X_{\lambda}$ is a nonempty set satisfying (i) and (ii). Then there exists $\bar{u} \in H_{0}^{1}(0,1) \cap H^{2}(0,1)$ being a solution of (1) such that

$$
\inf _{u \in X_{\lambda}} J_{\lambda}(u) \geq J_{\lambda}(\bar{u})
$$

Proof. Let us consider a minimizing sequence $\left(u_{m}\right)_{m \in N} \subset X_{\lambda}$ of $J_{\lambda}$. Without loss of generality we can assume that $J_{\lambda}\left(u_{m}\right) \leq M$ for $M>0$ sufficiently large. Now applying (10) we obtain

$$
M \geq J_{\lambda}\left(u_{m}\right)=\int_{0}^{1}\left(\frac{1}{2}\left|u_{m}^{\prime}(t)\right|^{2}-H\left(t, u_{m}(t), \lambda\right)\right) d t \geq-d \int_{0}^{1} \max _{u \in[0, d]}|f(t, u)+\lambda g(t, u)| d t .
$$

Thus, we have the boundedness of $\left(u_{m}^{\prime}\right)$ in $L^{2}(0,1)$, and further the boundedness of $\left(u_{m}\right)_{m \in N}$ in $H_{0}^{1}(0,1)$. Therefore $\left(u_{m}\right)_{m \in N}$ (up to a subsequence) is weakly convergent to $\bar{u} \in H_{0}^{1}(0,1)$, and as a result, by the Rellich-Kondrashov theorem, $\left(u_{m}\right)_{m \in N}$ tends uniformly to $\bar{u}\left(u_{m} \underset{m \rightarrow \infty}{\rightrightarrows} \bar{u}\right)$ in $[0,1]$. Moreover $\bar{u} \in C([0,1]), \bar{u}(0)=\bar{u}(1)=0$ and $0 \leq \bar{u} \leq d$ on $[0,1]$.

Our task is now to prove that $\bar{u}$ is the solution of (1). To this effect we apply Theorem 5 to guarantee the existence of the sequence $\left(v_{m}\right)_{m \in N} \subset H_{0}^{1}(0,1)$ such that

$$
-v_{m}^{\prime}(t)=f\left(t, u_{m}(t)\right)+\lambda g\left(t, u_{m}(t) \text { a.e. in }(0,1)\right.
$$

and

$$
\left.\lim _{m \rightarrow \infty} \int_{0}^{1} \frac{1}{2}\left|v_{m}(t)\right|^{2}+\frac{1}{2}\left|u_{m}^{\prime}(t)\right|^{2}-u_{m}^{\prime}(t) v_{m}(t)\right) d t=0
$$

Due to (17)

$$
\left|v_{m}^{\prime}(t)\right| \leq \max _{u \in[0, d]}|f(t, u)+\lambda g(t, u)| \text { a.e. in }(0,1)
$$


Since $\max _{u \in[0, d]}|f(t, u)+\lambda g(t, u)|$ belongs to $L^{2}(0,1)$ (assumption (H2)) we can see that $\left(v_{m}^{\prime}\right)_{m \in N}$ is bounded in $L^{2}(0,1)$. Taking into account the boundedness of $\left(u_{m}\right)_{m \in N}$ in $H_{0}^{1}(0,1)$ and $(18)$ we see that $\left(v_{m}\right)_{m \in N}$ is bounded in $L^{2}(0,1)$ which gives the weak convergence of $\left(v_{m}\right)_{m \in N}$ (up to a subsequence) in $L^{2}(0,1)$.

Finally, $\left(v_{m}\right)_{m \in N}$ is weakly convergent also in $H^{1}(0,1)$. Applying again the RellichKondrashov theorem, we see that $\left(v_{m}\right)_{m \in N}$ tends uniformly to $\bar{v} \in H^{1}(0,1)$. Now we can calculate

$$
\begin{aligned}
0 & \left.=\lim _{m \rightarrow \infty} \int_{0}^{1} \frac{1}{2}\left|v_{m}(t)\right|^{2}+\frac{1}{2}\left|u_{m}^{\prime}(t)\right|^{2}+u_{m}(t) v_{m}^{\prime}(t)\right) d t \\
& \left.\geq \int_{0}^{1} \frac{1}{2}|\bar{v}(t)|^{2}+\frac{1}{2}\left|\bar{u}^{\prime}(t)\right|^{2}+\bar{u}(t) \bar{v}^{\prime}(t)\right) d t \geq 0
\end{aligned}
$$

Thus

$$
\bar{v}(t)=\bar{u}^{\prime}(t) \text { a.e. in }(0,1) .
$$

From (17), we can use the weak convergence of $\left(v_{m}\right)_{m \in N}$ and the uniform convergence of $\left(u_{m}\right)_{m \in N}$ to obtain the following chain of equalities:

$$
\begin{aligned}
\int_{0}^{1} \bar{u}^{\prime}(t) h^{\prime}(t) d t & =\int_{0}^{1} \bar{v}(t) h^{\prime}(t) d t=\lim _{m \rightarrow \infty} \int_{0}^{1} v_{m}(t) h^{\prime}(t) d t=-\lim _{m \rightarrow \infty} \int_{0}^{1} v_{m}^{\prime}(t) h(t) \\
& =\lim _{m \rightarrow \infty} \int_{0}^{1}\left(f\left(t, u_{m}(t)\right)+\lambda g\left(t, u_{m}(t)\right)\right) h(t) d t \\
& =\int_{0}^{1}(f(t, \bar{u}(t))+\lambda g(t, \bar{u}(t))) h(t) d t
\end{aligned}
$$

for all $h \in H^{1}(0,1)$, where the last inequality is due to (H2) and the Lebesgue'adominated theorem. Now the du Bois-Reymond lemma yields that there exists $\bar{u}^{\prime \prime}$ a.e. in $(0,1)$ and

$$
-\bar{u}^{\prime \prime}(t)=f(t, \bar{u}(t))+\lambda g(t, \bar{u}(t)
$$

for a.e. $t \in(0,1)$. By the second part of $(\mathrm{H} 2), \bar{u}$ is the nontrivial solution.

As a consequence of the previous theorem and examples (1) and (3) we get the following.

Corollary 7. Assume hypotheses (H1)-(H3) (or (H4)). Then there exists $\bar{u} \in$ $X_{\lambda}:=\left\{u \in H_{0}^{1}(0,1) \cap H^{2}(0,1), 0 \leq u(t) \leq d\right.$ in $\left.[0,1]\right\}$ (or respectively $\bar{u} \in X_{\lambda}:=\{u \in$ $H_{0}^{1}(0,1) \cap H^{2}(0,1),|u(t)| \leq d$ in $\left.\left.[0,1]\right\}\right)$ being a nontrivial solution of (1) such that

$$
\inf _{u \in X_{\lambda}} J_{\lambda}(u)=J_{\lambda}(\bar{u}) .
$$

COROLlaRY 8. Under the assumptions (H1)-(H3) we have $0<\bar{u}(t)$ in $(0,1)$.

Proof. Suppose the contrary, that there exists $t_{0} \in(0,1)$ such that $\bar{u}\left(t_{0}\right)=0$. Since $\bar{u}(0)=\bar{u}(1)=0$ and $\bar{u} \in C^{1}(0,1) \cap C([0,1])$, Rolle's theorem leads to the existence of $0<\underline{t}_{1}<t_{0}<\bar{t}_{1}<1$ such that $\bar{u}^{\prime}\left(\underline{t}_{1}\right)=\bar{u}^{\prime}\left(\bar{t}_{1}\right)=0$. Moreover, by the fact that $\bar{u}^{\prime \prime}(t) \leq 0$ a.e. in $(0,1)$, we note that $\bar{u}^{\prime}$ is nonincreasing in $(0,1)$. Thus, assertion $\bar{u}^{\prime}\left(\underline{t}_{1}\right)=\bar{u}^{\prime}\left(\bar{t}_{1}\right)=$ 
0 implies $\bar{u}^{\prime}(t)=0$ for all $t \in\left[t_{1}, \bar{t}_{1}\right]$. It is clear that applying again Rolle's theorem we can derive the existence of $\underline{t}_{2}$ and $\bar{t}_{2}$ such that $0<\underline{t}_{2}<\underline{t}_{1}<t_{0}<\bar{t}_{1}<\bar{t}_{2}<1$ and $\bar{u}^{\prime}\left(t_{2}\right)=\bar{u}^{\prime}\left(\bar{t}_{2}\right)=0$, which gives $\bar{u}^{\prime}(t)=0$ for all $t \in\left[\underline{t}_{2}, \bar{t}_{2}\right]$. Iterating this process we can construct sequences $\left(\underline{t}_{m}\right)_{m=1}^{\infty}$ and $\left(\bar{t}_{m}\right)_{m=1}^{\infty}$ such that $\bar{u}^{\prime}(t)=0$ for all $t \in\left[\underline{t}_{m}, \bar{t}_{m}\right]$ and $\bigcup_{m=1}^{\infty}\left[t_{m}, \bar{t}_{m}\right]=(0,1)$, so that $\bar{u}(t)=$ const in $(0,1)$. Finally, by the boundary condition and the continuity of $\bar{u}$, we note that $\bar{u}(t)=0$ in $(0,1)$. This contradicts the fact that $\bar{u}$ is nontrivial solution of our problem.

5. Continuous dependence of solutions on functional parameters. Now we investigate the Dirichlet problem

$$
\begin{aligned}
& -u^{\prime \prime}=f(t, u, v)+\lambda g(t, u, v) \text { a.e. in }(0,1) \\
& u(0)=u(1)=0
\end{aligned}
$$

where $v \in V \subset L^{2}(0,1)$, under the following assumptions:

(H1v) There exists $d_{v}>0$ such that $f, g:(0,1) \times\left(-\varepsilon, d_{v}+\varepsilon\right) \times R \rightarrow R$ are Caratheodory functions, $f+\lambda g$ is nondecreasing with respect to the second variable in $(-\varepsilon, d+\varepsilon), \varepsilon$ is given positive number.

$(\mathrm{H} 2 \mathrm{v})$ For all $v \in V, \max _{u \in[0, d]}|f(t, u, v)+\lambda g(t, u, v)|$ belongs to $L^{2}(0,1) ; f(t, 0, v(t))+$ $\lambda g(t, 0, v(t)) \neq 0$.

Since we will consider the case when $X_{\lambda}:=\left\{u \in H_{0}^{1}(0,1) \cap H^{2}(0,1),|u(t)| \leq d\right.$ in $[0,1]\}$ or $X_{\lambda}:=\left\{u \in H_{0}^{1}(0,1) \cap H^{2}(0,1), 0<u(t) \leq d\right.$ in $\left.[0,1]\right\}$ we need the following additional assumptions:

(H3v) For all $v \in V, f+\lambda g \geq 0$ in $(0,1) \times\left[0, d_{v}\right] \times R$ and the following estimations hold

$$
0<\int_{0}^{1}\left(f\left(t, d_{v}, v(t)\right)+\lambda g\left(t, d_{v}, v(t)\right)\right) d t \leq 4 d_{v}
$$

or

$$
0<\int_{0}^{1} t(1-t)\left(f\left(t, d_{v}, v(t)\right)+\lambda g\left(t, d_{v}, v(t)\right)\right) d t \leq d_{v} .
$$

$(\mathrm{H} 4 \mathrm{v})$ For all $v \in V$, there exists $0<d_{v} \leq d$ such that

$$
\int_{0}^{1} \max _{u \in\left[0, d_{v}\right]}|f(t, u, v(t))+\lambda g(t, u, v(t))| d t \leq 4 d_{v}
$$

or

$$
\int_{0}^{1} t(1-t) \max _{u \in\left[0, d_{v}\right]}|f(t, u, v(t))+\lambda g(t, u, v(t))| d t \leq d_{v} .
$$

(H5v) There exists $d>0$ such that for all $v \in V, d_{v} \leq d$.

TheOrem 9. Assume (H1v)-(H2v), (H4v) (or (H3v) for positive solutions) and (H5v) and suppose that the sequence of parameters $\left\{v_{m}\right\}_{m=1}^{\infty} \subset V$ is convergent to $v_{0} \in V$ a.e. in $(0,1)$. Let us denote by $u_{m}$ a solution (positive if (H3v) holds) of (19) with $v=v_{m}, m=1,2, \ldots$, such that $u_{m}(t) \leq d_{v_{m}}$ for all $t \in(0,1)$. (Their existence follows form Corollary 7.) Then there exists a subsequence, still denoted by $\left\{u_{m}\right\}_{m=1}^{\infty}$, 
uniformly convergent to $u_{0}$ which is a solution (positive if (H3v) holds) of (19) with $v=v_{0}$ and $u_{0}(t) \leq d$ for all $t \in(0,1)$. Also we have

$$
u_{0}(t)=O(1-t) \quad \text { for } t \rightarrow 1^{-}
$$

and

$$
u_{0}(t)=o(\phi(t)) \quad \text { for } t \rightarrow 1^{-},
$$

for all functions $\phi \in C^{1}(0,1)$ such that $\lim _{t \rightarrow 1^{-}} \phi(t)=0$ and $\lim _{t \rightarrow 1^{-}} \phi^{\prime}(t)=+\infty$.

Proof. We start our proof with the observation that

$$
\begin{aligned}
\int_{0}^{1}\left(u_{m}^{\prime}(t)\right)^{2} d t & =-\int_{0}^{1} u_{m}^{\prime \prime}(t) u_{m}(t) d t \\
& =\int_{0}^{1} f\left(t, u_{m}, v_{m}(t)\right)+\lambda g\left(t, u_{m}, v_{m}(t)\right) u_{m}(t) d t \leq 4 d^{2},
\end{aligned}
$$

which follows from the fact that $u_{m}$ satisfies (19) a.e. in $(0,1)$ (with $\left.v_{m}\right)$ and $\left|u_{m}(t)\right| \leq d$ in $[0,1]$. Thus $\left\{u_{m}^{\prime}\right\}_{m=1}^{\infty}$ is bounded in $L^{2}(0,1)$, and further $\left\{u_{m}\right\}_{m=1}^{\infty}$ (up to a subsequence) is weakly convergent in $H_{0}^{1}(0,1)$ to a certain $u_{0} \in H_{0}^{1}(0,1)$. Applying the RellichKondrashov theorem we see that $\left\{u_{m}\right\}_{m=1}^{\infty}$ is uniformly convergent to $u_{0}$ in $[0,1]$. Consequently $u_{0} \in C([0,1]),\left(u_{m} \in C([0,1])\right)$. This implies $0 \leq u_{0}(t) \leq d \in$ for $t \in[0,1]$ and $u_{0}(0)=u_{0}(1)=0$. Our task is now to show that $u_{0}$ is a solution of (19) with $v=v_{0}$. To this end we calculate for each $h \in H_{0}^{1}(0,1)$

$$
\begin{aligned}
\int_{0}^{1} u_{0}^{\prime}(t) h^{\prime}(t) d t & =\lim _{m \rightarrow \infty} \int_{0}^{1} u_{m}^{\prime}(t) h^{\prime}(t) d t=\lim _{m \rightarrow \infty} \int_{0}^{1}\left(-u_{m}^{\prime \prime}(t) h(t)\right) d t \\
& =\lim _{m \rightarrow \infty} \int_{0}^{1} f\left(t, u_{m}, v_{m}(t)\right)+\lambda g\left(t, u_{m}, v_{m}(t)\right) h(t) d t
\end{aligned}
$$

Taking into account $(\mathrm{H} 2 \mathrm{v})$ and using the Lebesgue'a dominated theorem one sees that

$$
\begin{aligned}
& \lim _{m \rightarrow \infty} \int_{0}^{1} f\left(t, u_{m}(t), v_{m}(t)\right)+\lambda g\left(t, u_{m}(t), v_{m}(t)\right) h(t) d t \\
& =\int_{0}^{1} f\left(t, u_{0}(t), v_{0}(t)\right)+\lambda g\left(t, u_{0}(t), v_{0}(t)\right) h(t) d t
\end{aligned}
$$

Finally, for each $h \in H_{0}^{1}(0,1)$ the following equality holds

$$
\int_{0}^{1} u_{0}^{\prime}(t) h^{\prime}(t) d t=\int_{0}^{1} f\left(t, u_{0}(t), v_{0}(t)\right)+\lambda g\left(t, u_{0}(t), v_{0}(t)\right) h(t) d t
$$

Now, the du Bois-Reymond Lemma leads to the conclusion that

$$
-u^{\prime \prime}(t)=f\left(t, u_{0}(t), v_{0}(t)\right)+\lambda g\left(t, u_{0}(t), v_{0}(t)\right) \text { a.e. in }(0,1) .
$$

Summarizing, $u_{0} \in H_{0}^{1}(0,1) \cap H^{2}(0,1), 0 \leq u_{0}(t) \leq d$ in [0,1] and satisfies (19) a.e. in $(0,1)$ in the case when $v=v_{0}$. As in Corollary 8 , we can show $0<u_{0}(t)$ for all $t \in(0,1)$. 
Employing the approach similar to that in the paper [3] we investigate the asymptotic behaviour of $u_{0}$. To this end we have to note that $u_{0}$ satisfies

$$
u_{0}(t)=\int_{0}^{1} G(t, s)\left(f\left(s, u_{0}(s)\right)+\lambda g\left(s, u_{0}(s)\right)\right) d s .
$$

Thus

$$
\begin{gathered}
\int_{0}^{1} s\left(f\left(s, u_{0}(s)\right)+\lambda g\left(s, u_{0}(s)\right)\right) d s=-\lim _{t \rightarrow 1^{-}} u_{0}^{\prime}(t)=\lim _{t \rightarrow 1^{-}} \frac{u_{0}(t)}{1-t} \\
0=\lim _{t \rightarrow 1^{-}} \frac{u_{0}^{\prime}(t)}{\varphi^{\prime}(t)}=\lim _{t \rightarrow 1^{-}} \frac{u_{0}(t)}{\varphi(t)}
\end{gathered}
$$

for each $\phi \in C^{1}(0,1)$ such that $\lim _{t \rightarrow 1^{-}} \phi(t)=0$ and $\lim _{t \rightarrow 1^{-}} \phi^{\prime}(t)=+\infty$.

EXAMPLE 10. For each $m=1,2, \ldots$, the Dirichlet problem

$$
\begin{aligned}
-u^{\prime \prime}(t) & =\frac{1}{8 \sqrt[4]{t}}(u(t))^{5}+\lambda\left(1+\frac{t}{1+m^{2} t^{2}}\right) \\
u(0) & =u(1)=0,
\end{aligned}
$$

with $0<\lambda \leq \frac{8}{6}$, possesses at least one positive solution $u_{m}$ such that $u_{m}(t) \leq 2$. Moreover, there exists at least one positive solution $u_{0}$ for the limit problem

$$
\begin{aligned}
-u^{\prime \prime}(t) & =\frac{1}{8 \sqrt[4]{t}}(u(t))^{5}+\lambda \\
u(0) & =u(1)=0,
\end{aligned}
$$

such that $\left(u_{m}\right)_{m}$ tends uniformly (up to a subsequence) to $u_{0}$ in $[0,1]$.

Proof. It is easy to note that for each $v \in V:=\left\{1+\frac{t}{1+m^{2} t^{2}}, m=1,2, \ldots\right\} \cup\{1\}$ the functions

$$
f(t, u, v)=\frac{1}{8 \sqrt[4]{t}}(u(t))^{5} \text { and } g(t, u, v)=v
$$

satisfy assumptions ( $\mathrm{H} 1 \mathrm{v})-(\mathrm{H} 2 \mathrm{v})$ and $(\mathrm{H} 5 \mathrm{v})$ with $d_{v}=d=2, \varepsilon=0.1$ and $f+\lambda g$ is increasing with respect to the second variable. Thus, we have to show only that one of the inequalities from (H3) also holds. To this end we note

$$
\begin{aligned}
& \int_{0}^{1} \max _{u \in[0,2]}\left[\frac{1}{8 \sqrt[4]{t}}(u(t))^{5}+\lambda\left(1+\frac{t}{1+m^{2} t^{2}}\right)\right] d t \\
& \leq \int_{0}^{1} \frac{4}{\sqrt[4]{t}} d t+2 \lambda \leq 8
\end{aligned}
$$

Now taking into account the fact that $\left(1+\frac{t}{1+m^{2} t^{2}}\right)_{m=1}^{\infty}$ tends uniformly to 1 in $[0,1]$, we can apply Theorem 9 and see that the sequence $\left(u_{m}\right)_{m}$ of solutions (20) tends uniformly (up to a subsequence) to $u_{0}$ in $[0,1]$, where $u_{0}$ is positive bounded solution for (21) 
EXAMPLE 11. Let us consider the sequence of problems

$$
\begin{aligned}
-u^{\prime \prime}(t) & =\frac{a(t)(\sin t)^{8}}{\sqrt[4]{t}}\left(e^{u(t)}+\lambda \frac{(u(t))^{4}(\cos t)^{2}}{(2-u(t))(3+u(t))}\right) \sqrt{\frac{\operatorname{arctg}^{2}(t)}{m^{2}}+\sqrt[4]{t}} \text { a.e. in }(0,1) \\
u(0) & =u(1)=0
\end{aligned}
$$

for all $m=1,2, \ldots, 0<\lambda<35.36$ and $a \in L^{\infty}(0,1), 0<a(t)<1$ for a.e. $t \in(0,1)$. Then for each $m$ there exists a positive solution $u_{m}$ such that $u_{m}(t) \leq 1$, 5. Moreover there exists at least one positive solution $u_{0}$ for the limit problem

$$
\begin{aligned}
& -u^{\prime \prime}=a(t) \frac{(\sin t)^{8}}{\sqrt[4]{t}}\left(e^{u}+\lambda \frac{u^{4}(\cos t)^{2}}{(2-u)(3+u)}\right) \sqrt[8]{t} \text { a.e. in }(0,1) \\
& u(0)=u(1)=0
\end{aligned}
$$

such that $\left(u_{m}\right)_{m}$ tends uniformly (up to a subsequence) to $u_{0}$ in $[0,1]$.

Proof. Let $V:=\left(v_{m}\right)_{m=1}^{\infty} \cup\left\{v_{0}\right\}$, with

$$
v_{m}=\sqrt{\frac{\operatorname{arctg}^{2}(t)}{m^{2}}+\sqrt[4]{t}} \text { and } v_{0}=\sqrt[8]{t} .
$$

We start the proof with the observation that $\left(v_{m}\right)_{m=1}^{\infty}$ converges uniformly to $v_{0}$ in $[0,1]$. Our task is now to show that for each $v \in V,(\mathrm{H} 1 \mathrm{v})-(\mathrm{H} 3 \mathrm{v})$ and $(\mathrm{H} 5 \mathrm{v})$ hold with $d_{v}=d=1.5$. First we note that

$$
f(t, u, v)=a(t) \frac{(\sin t)^{8}}{\sqrt[4]{t}} e^{u} v \text { and } g(t, u, v)=a(t) \frac{(\sin t)^{8}}{\sqrt[4]{t}} \frac{u^{4}(\cos t)^{2}}{(2-u)(3+u)} v
$$

are measurable in $t$ and continuous with respect to the pair $(u, v)$ in $[-0.1,1.6] \times R$.

Since for each $v \in V$

$$
\begin{aligned}
& \frac{d}{d u}((f(t, u, v)+\lambda g(t, u, v))) \\
& \quad=\frac{a(t)(\sin t)^{8}}{\sqrt[4]{t}}\left(e^{u}+\lambda(\cos t)^{2} u^{3} \frac{\left(-2 u^{2}-3 u+24\right)}{\left(-u^{2}-u+6\right)^{2}}\right) v>0
\end{aligned}
$$

for $(t, u) \in(0,1) \times(-0.1,1.6)$, we see that $f(t, u, v)+\lambda g(t, u, v)$ is increasing with respect to $u$ in $(-0.1,1.6)^{6}$. To show that for all $v \in V, t \mapsto \max _{u \in[0, d]} \mid f(t, u, v(t))+$ $\lambda g(t, u, v(t)) \mid$ belongs to $L^{2}(0,1)$, it suffices to use the estimation

$$
\begin{aligned}
\max _{u \in[0, d]}|f(t, u, v(t))+\lambda g(t, u, v(t))| & =f(t, d, v(t))+\lambda g(t, d, v(t)) \\
& \leq\left(\frac{9}{4} \lambda+e^{\frac{3}{2}}\right) \sqrt{\frac{1}{4} \pi^{2}+1} \frac{1}{\sqrt[4]{t}} .
\end{aligned}
$$

Finally we get $(\mathrm{H} 1 \mathrm{v})-(\mathrm{H} 2 \mathrm{v})$ satisfied. Our task is now to prove that $(\mathrm{H} 3 \mathrm{v})$ follows. To this end we note, by (24), that for all $v \in V, f+\lambda g \geq 0$ in $(0,1) \times[0,1.5] \times R$. 
Moreover for all $m=1,2, \ldots$

$$
\begin{aligned}
& \int_{0}^{1} \max _{u \in[0, d]}\left(f\left(t, u, v_{m}(t)\right)+\lambda g\left(t, u, v_{m}(t)\right)\right) d t \\
& \leq \int_{0}^{1} \frac{(\sin t)^{8}}{\sqrt[4]{t}}\left(e^{u}+\lambda \frac{u^{4}(\cos t)^{2}}{(2-u)(3+u)}\right) \sqrt{\frac{\operatorname{arctg}^{2}(t)}{m^{2}}+\sqrt[4]{t}} d t \\
& \leq \sqrt{\frac{\pi^{2}}{4 m^{2}}+1} \int_{0}^{1} \frac{(\sin t)^{8}}{\sqrt[4]{t}}\left(e^{\frac{3}{2}}+\lambda \frac{\left(\frac{3}{2}\right)^{4}}{\left(\frac{1}{2}\right)\left(\frac{9}{2}\right)}\right) d t \\
& \leq \sqrt{\frac{\pi^{2}}{4}+1}\left(e^{\frac{3}{2}}+\lambda \frac{\left(\frac{3}{2}\right)^{4}}{\left(\frac{1}{2}\right)\left(\frac{9}{2}\right)}\right) \int_{0}^{1} \frac{(\sin t)^{8}}{\sqrt[4]{t}} d t \\
& =0.16062 \lambda+0.31993<6 .
\end{aligned}
$$

In the same way we see that

$$
\int_{0}^{1} \max _{u \in[0, d]}\left(f\left(t, u, v_{0}(t)\right)+\lambda g\left(t, u, v_{0}(t)\right)\right) d t \leq 6 .
$$

Finally, we see that the assumptions of Theorem 9 are satisfied. Thus we derive that $\left(u_{m}\right)_{m}$ tends uniformly (up to a subsequence) to $u_{0}$ in $[0,1]$, where $u_{0}$ is positive bounded solution for (23).

\section{REFERENCES}

1. R. P. Agarwal and D. O'Regan, Singular differential and integral equations with applications (Kluwer Academic Publisher, Dordrecht, 2003).

2. R. P. Agarwal and D. O'Regan, Twin solutions to singular Dirichlet problems, J. Math. Anal. Appl. 240 (1999), 433-445.

3. S. Djebali and A. Orpel, A note on positive evanescent solutions for a certain class of elliptic problems, J. Math. Anal. Appl. 353 (2009), 215-223.

4. A. Nowakowski and A. Rogowski, Multiple positive solutions for a nonlinear Dirichlet problem with nonconvex vector-valued response, Proc. R. Soc. Edinburgh 135A (2005), 105-117.

5. D. O'Regan, Theory of singular boundary value problems (World Scientific, Singapore, 1994).

6. A. Orpel, On the existence of bounded positive solutions for a class of singular BVP, Nonlinear Anal. 69 (2008), 1389-1395. 1522.

7. A. Orpel, Nonlinear BVPS with functional parameters, J. Differ. Equ. 246 (2009), 1500

8. H. Lü, D. O'Regan and R. P. Agarwal, An approximation approach to eigenvalue intervals for singular boundary value problems with sign changing nonlinearities, Math. Inequalities Appl. 11 (2007), 81-98.

9. H. Lü, D. O'Regan and R. P. Agarwal, Existence to singular boundary value problems with sign changing nonlinearities using an approximation methods approach, Appl. Math. 52 (2007), 117-135. 